\title{
Evaluation of primary mental health care in North West province - a qualitative view
}

Claire van Deventer, MB ChB, FCFP, MFamMed (Med), BA

Family physician, Dr Kenneth Kaunda District, North West, and Department of Family Medicine, University of the Witwatersrand

Ian Couper, MB BCh, MFamMed (Med), BA

Rural Health, Department of Family Medicine, University of the Witwatersrand and North West Province Department of Health

Anne Wright, MA (Sociol), PhD

Department of Family Medicine, University of the Witwatersrand

John Tumbo, MB ChB, MCFP, MFamMed (Med)

Department of Family Medicine and Primary Care, University of Limpopo and North West Province Department of Health

Charles Kyeyune, MB ChB, MFamMed (Med), DTM\&H, $\mathrm{DPH}, \mathrm{MBA}$

Rural Health, Department of Family Medicine, University of the Witwatersrand and North West Province Department of Health

Background. Owing to many complaints by health care workers and patients and a perceived poor standard of care, the mental health care services in North West province were assessed using quantitative and qualitative methods. The Mental Health Care Act of 2002 makes provision for the integration of services into primary health care. Previously, dedicated mental health care co-ordinators were doing the bulk of the management of this category of patients.

Methodology. The qualitative part of the study consisted of free attitude interviews with professional nurses at clinics and focus group interviews with patients, caregivers and mental health care co-ordinators.

Results. There was general satisfaction with the current services. Some dissatisfaction was expressed regarding issues of individualised care versus integration into the general primary care services. Concerns were expressed about resource constraints - in terms of human and physical resources, communication, training, and the role of specialised care.

Conclusion. This study highlights issues around integration of mental health care services into primary care, and has provided information for managers and clinicians to utilise in the improvement of mental health care.

Effective community-based mental health care as part of primary care is essential for the integration of mental health patients into society. In South Africa, the Mental Health Care Act of 2002 makes provision for the integration of mental health care into state services at primary care level. Previously, in many provinces dedicated mental health care co-ordinators were doing the bulk of the management of this category of patients. The integration of mental health care services has led to mental health care users becoming part of the general patient load in clinics. Mental health care users as well as professionals have been dissatisfied about the difficulties inherent in changing a system.

Even before these changes, however, a survey of psychiatric community services in one sub-district in North West province in 2000/1 indicated lack of consistency in the assessment of mentally ill patients, little dedicated mental health care or medical input, and lack of proper record keeping

The issues surrounding the management of mentally ill patients are complex. Lehman and Zastowny ${ }^{2}$ found in their meta-analysis that patients often express high rates of satisfaction with their mental health care, and that there is a lack of controlled studies on patient satisfaction. They also found that chronic patients expressed less satisfaction with their treatment than non-chronic patients.

In spite of this, there have been South African studies pointing to important causes of dissatisfaction. In a study using free attitude interviews in the Mmamethake Health District, Limpopo province, Shariff ${ }^{3}$ noted the problems of disorganised service delivery; this was highlighted by all mentally ill patients interviewed, who expressed anger and frustration. Patients also complained about a paternalistic approach on the part of nurses, to the extent of being given injections against their will, and non-involvement of themselves and/or their families in treatment. Poor communication between the different levels of care was also highlighted. 
We know from previous research ${ }^{3,4}$ that users of mental health services in South Africa are unhappy, but it is not clear what they believe would change their situation. Furthermore, we do not know how health care providers themselves perceive this problem, or what they think could be done to improve it.

The aim of the current research was to evaluate clinic-based mental health services in all four sub-districts in North West province, using quantitative and qualitative methods. The main finding of the quantitative part of the study was the extremely poor documentation regarding mentally ill patients as well as an inadequate system of review by doctors and nurses. ${ }^{4}$ The qualitative results are presented in this article.

\section{Methods}

The design was a cross-sectional qualitative study using focus group interviews with patients and mental health co-ordinators and individual depth interviews with clinic staff.

A random sample of 16 facilities was taken out of all clinics and community health centres in the North West province, stratified into the four districts within the province. One health centre together with three functioning clinics were included from each district.

For the purposes of this study, mental health patients were defined as those suffering both from chronic major psychiatric conditions such as schizophrenia, depression, bipolar disorder, dementia, etc., and from acute common problems such as acute anxiety, adjustment disorders, etc. Epilepsy, which is commonly (and incorrectly) defined in primary care clinics as a mental health problem, was excluded unless there was a concomitant psychiatric diagnosis (e.g. epileptic psychosis). Patients could have more than one diagnosis. Common problems requiring counselling such as spouse abuse, HIV, sexual difficulties, etc. were excluded. Mental retardation, as a primary diagnosis, was also excluded.

Five focus group discussions were conducted with patients and caregivers. The researchers sought to obtain a range of responses through the mix of patients (both in terms of demographics and their conditions). The focus group discussions were conducted by trained research assistants from the Madibeng Centre for Research. The initial exploratory question posed was, 'What do you feel about the treatment and care that you receive at this clinic for your problem?' Once saturation was reached, a follow-up question was asked, 'What do you think can be done to improve the care that you receive at this clinic?' The focus group interviews were conducted in Setswana and Afrikaans and audiotaped. The interviews were transcribed and, in the case of the Setswana ones, translated into English for the purpose of data analysis.

Five professional nurses in three of the districts (Bojanala, Southern and Bophirima) were interviewed using free attitude interview techniques. The initial exploratory question asked was, 'What do you feel about managing mentally ill patients in your clinic?' A follow-up question was asked, namely 'What do you think can be done to improve the management of these patients in your clinic?'

Three doctors, each of whom was the allocated clinic doctor at one of the clinics visited in the above-mentioned districts, were interviewed using the same questions.

At a meeting of the mental health co-ordinators from all the subdistricts in the province, eight volunteers were invited to join a focus group interview. The group came from all districts and population groups in the province. The process was conducted in a 'fish-bowl' arrangement, with the focus group in the middle of the room and the other co-ordinators observing the process. The initial exploratory question was, 'What do you feel about the management of mentally ill patients in your sub-district?' $A$ follow-up question was asked, 'What do you think can be done to improve the management of these patients in your sub-district?'

The interviews were conducted during 2005. Transcriptions of the interviews were used for data analysis. Content analysis was initially carried out in order to develop lists of issues emerging from each set of data, individually and then together (the patients and caregivers, the professional nurses and doctors, and the mental health co-ordinators respectively).

The study received ethical approval from the Human Research Ethics Committee of the University of the Witwatersrand.

The informed consent of all people interviewed, individually and in focus groups, was obtained. No names or identifying details of patients, staff or clinics are used in reporting the results.

\section{Results}

Four clinics were used for the focus groups. In total, 35 patients and caregivers participated. In two groups, the caregivers joined the patients as there were only one or two caregivers available.

The two major themes were general satisfaction, mostly from the patient themselves, but dissatisfaction regarding specific issues such as communication problems, lack of continuity and long 
waiting times (Table I). Patients and their caregivers expressed the desire to be seen separately and indicated a need for more doctors. There was therefore a balance of satisfaction, dissatisfaction and doubts, as represented in Fig. 1.

A range of other opinions were expressed about specific issue, e.g. the effect of money, education, etc.

Eight interviews were conducted with professional nurses in the clinics and doctors visiting these clinics.

The main discussion centred on dedicated specialised mental health services versus integration into so-called supermarket services. The commonly held view was that primary health nurses and doctors do not have the expertise needed to manage mental health patients. In areas where there were dedicated services, these were seen as providing much more effective care than the integrated services. Experienced professional nurses said 'You have to individualise care', and 'A psychiatric guy is not someone who can sit in a queue'. Other concerns raised included the lack of a psychiatric unit, the absence of specialists, rapid discharge from referral hospitals leading to relapse, ignorance of the Mental Health Care Act and the possibility of patient abuse. Fig. 2 presents a summary of the issue.

One focus group discussion was conducted with eight mental health co-ordinators. The words most often heard in this group were 'lack of' and 'problem' (Fig. 3). Most of the problems

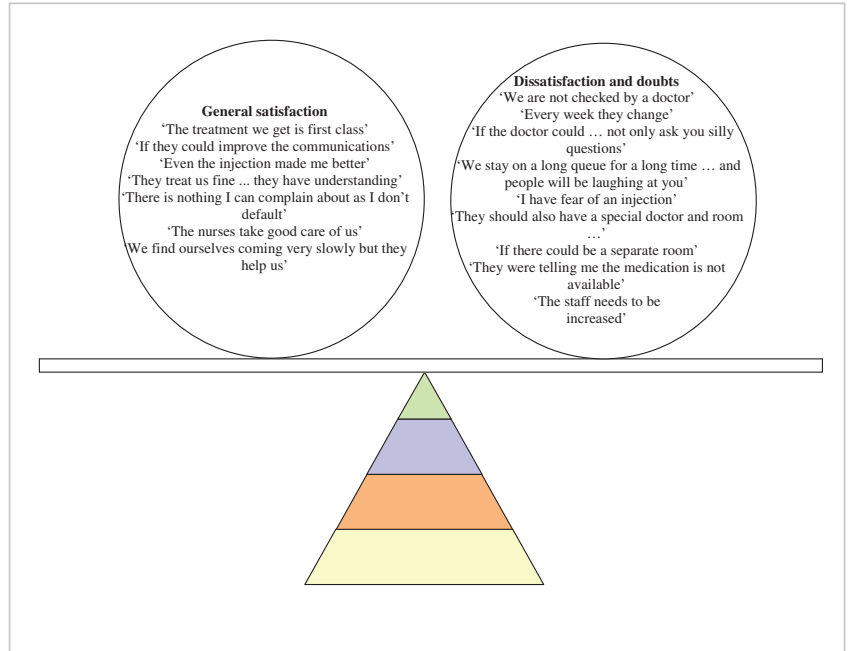

Fig. 1. Satisfaction versus dissatisfaction with mental health care.

related to resources, either human resources such as lack of specialised and knowledgeable personnel, or physical resources, such as lack of transport for home visits, absence of institutions for children, and inadequate medication depending on the level of care. Training was also discussed as a priority. Attitudes of colleagues, managers and leaders in the province towards mental health were deplored. Lack of teamwork, integration and co-ordination by professionals and community involvement were also identified as barriers to care. The Mental Health Care Act was touched on as a misunderstood entity.

Table I. Major and minor themes from patients and their caregivers

\section{Major themes}

General satisfaction

Dissatisfaction and doubts regarding certain areas

Minor themes
Patients felt they receive good treatment and the care was clearly of a high standard. There was recognition by some of the responsibility they themselves have in getting good care. Nurses were seen as being friendly and dedicated, and thus providing good care

\section{Communication}

Lack of continuity due to staff turnover

Long waiting times

A desire expressed for specialised services in the form of special clinics and dedicated staff

A need for more staff was noted

Particular need for doctors to be involved in their treatment

Care for physical problems should not be separated from mental health problems The involvement of staff members beyond simple treatment was appreciated, the example being given of a party for patients. Patients felt their family and social problems were being addressed. However, social workers would help this Caregivers believe family members are educated about the illness, but patients do not always see it the same way

Patients have special needs that must be considered

Money was raised as an important issue in respect of accessing treatment Some patients felt that everyone was in it for themselves with no support for each other, while others felt they did meet together and support each other The issue of stigma in the community was also raised - discrimination is a significant issue 


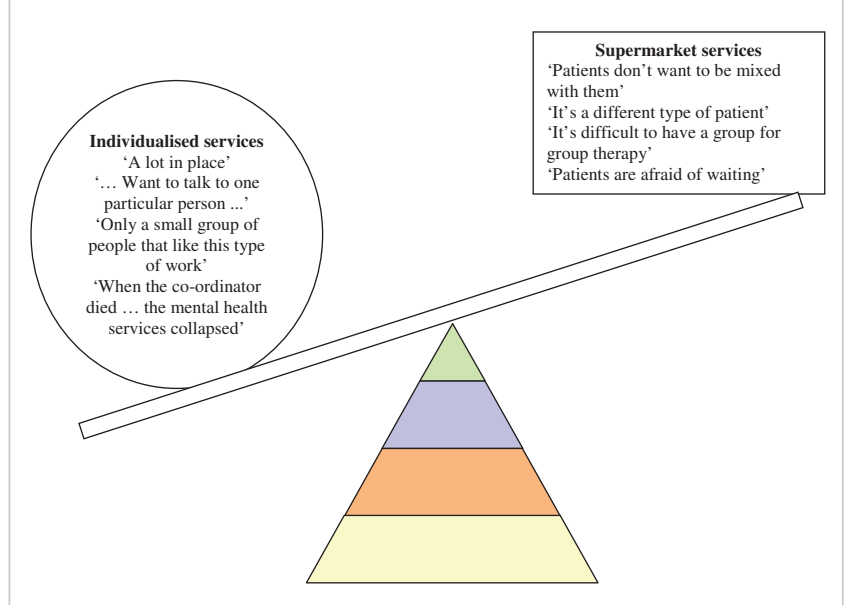

Fig. 2. Individualised versus supermarket approach in mental health care.

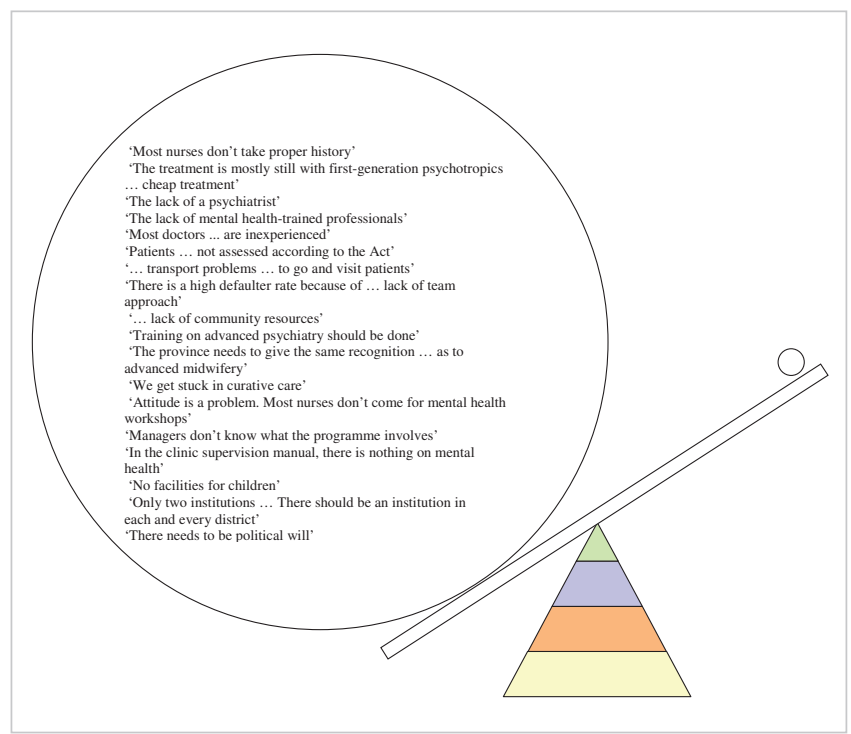

Fig. 3. Overwhelming systemic problems in mental health care.

\section{Discussion}

Despite the problems noted, it is encouraging that patients and their caregivers are generally satisfied and feel they receive a good standard of care, from nurses who are sincerely interested in their welfare. Modiba et al.' 'similarly found that a high proportion (77\%) of patients and caregivers in Moretele sub-district perceived the clinic service to be helpful. Staff members themselves feel they are generally giving good care to mental health patients. It is also very clear that nurses are the backbone of the service, and that doctors are quite peripheral. Any intervention that does not take this into account will not succeed.

However, important concerns were raised by all groups. Communication problems are one such issue. It is clear that patients are not always sure when they should come for followup, and often find the process confusing. Patients and caregivers struggle with a lack of continuity, seeing different staff members each time, and with the length of time spent waiting, both in the clinics and at hospital. To address these and other concerns, they plead for a specialised service for mentally ill patients. This is echoed by doctors and nurses, who argue that specialised services are needed due to the skills required, the difficulty of ensuring adherence to treatment, the importance of continuity, the long waiting times for patients, etc. There is a strong feeling among staff members generally that the supermarket approach is not appropriate for mental health care. There is however a mandate in the Mental Health Act that these services need to be integrated. That means that ways need to be found to empower staff and systems in order for mental health care users to receive the best of care.

Limited human resources featured strongly in all interviews and focus group discussions. According to patients there is insufficient staff, especially doctors. This is echoed by nurses and doctors. Mental health co-ordinators add that there are not only too few doctors, especially psychiatrists, but also too few psychologists, social workers and other members of the mental health care team, a lack also noted in the earlier study in Moretele.'

Various suggestions are made to deal with the problem. A range of models for offering mental health services need to be explored, such as the four main models currently being used in the National Health Service in the UK, viz. community mental health teams that provide increased liaison and crisis intervention; psychiatrists who operate clinics in health centres; community psychiatric nurses designated to work with those with mental health problems in a primary care setting; and provision of advice and skills to primary care teams from specialist mental health services. ${ }^{5}$ These need not necessarily oppose integration of services, but rather offer innovative ways to support and encourage the new paradigm.

Patients express a need for support - both support from the service and support from fellow sufferers. This highlights the need expressed for greater involvement in social issues, such as extending care into patients' homes (limited by both time and transport), the need for social workers and the lack of education of families and community members about mental illness. Modiba et al.' noted that community involvement was viewed by informants in Moretele sub-district as key to providing communitybased mental health services to people with mental illness.

There is much focus in the literature on the patient as the one to blame for 'non-compliance' in one way or another, either because of their disease or because of their responses to the 
medication they have to take, ${ }^{6-16}$ but there has been little focus on the health system as a possible cause of non-adherence. Yet our findings from these interviews, together with analysis of patient records, suggest a system in which there is every reason for nonadherence, from insufficient and unskilled staff, to inadequate or absent records, to poor communication and referral pathways, to poor prescribing, to irregular and inadequate reviews.

It is clear from the literature that, just as there is no scientific evidence favouring the use of hospital services alone for mental health care, there is also no evidence that community services alone can provide satisfactory and comprehensive care; ${ }^{17}$ both are needed, and finding ways of ensuring they work together and support each other is critical.

Swartz and MacGregor ${ }^{18}$ argue strongly against the idea that chronic psychiatric illness can be treated in the same way as other chronic illnesses in an overstretched and narrowly biomedically focused system. This may be true - and certainly the concerns raised in this study reveal the need for greater specialisation - but we would also argue that the problem is a broader one. Our experience is that chronic illnesses in general are not being managed well, and overhauling chronic illness care, through the introduction of sound principles and appropriate systems, including adequate training and record-keeping, would go a long way towards dealing with the problems in mental health care as well. This could address the problems raised here in a context of integration.

There were limitations to the study. The quality of some data is questionable, as the patient groups were small and the transcripts indicated that the discussions were not well-facilitated. Despite this, general trends and patterns could be observed.

\section{Conclusions}

Although based on a relatively small sample of participants, this research has highlighted important trends in the care of mentally ill people in North West province, which we believe can be generalised to other provinces. Despite general satisfaction being expressed by patients and even health care staff, there are crucial areas that need attention, such as how integration is managed in the context of limited resources, the role of specialised input and support, communication between different levels of care as well as between health workers and patients, and correct implementation of the Mental Health Care Act.
There is a need for more rigorous research in this arena, which would be able to highlight more accurately the discrepancies between policy imperatives and implementation processes.

Critical to the success of any intervention are active political backing and managerial support, taking the problems of mental health seriously and providing the necessary resources for this.

We wish to thank all the patients and staff who participated in the interviews, the research assistants from the Madibeng Centre for Research, Brits, who helped with the data collection, and the Directorate of Knowledge Management, North West Department of Health, for their support.

\section{References}

1. Modiba P, Porteus K, Schneider H, et al. Community Mental Health Service Needs: A Study of Service Users, Their Families, and Community Leaders in the Moretele District, North-West Province. Johannesburg: Centre for Health Policy, University of the Witwatersrand, 2000

2. Lehman AF, Zastowny TR. Patient satisfaction with mental health services: a metaanalysis to establish norms. Eval Program Plann 1983; 6(3-4): 265-274.

3. Shariff SA. Reasons for non-compliance with treatment among patients suffering from psychiatric illnesses in Mmamethake Health District. MFamMed Dissertation Medunsa, 2000.

4. Couper I, Wright A, van Deventer C, et al. Evaluation of primary mental health care in North West province (Research report). 2006. http:// www.madibeng.org.za/ reports. htm (accessed 30 June 2008).

5. Lester $H$, Glasby J, Tylee A. Integrated primary mental health care: threat or opportunity in the new NHS? Br J Gen Pract 2004; 5415011: 285-291.

6. Haynes RB. Determinants of compliance: The disease and the mechanics of treatment. In: Haynes RB, Taylor DW, Sackett DL, eds. Compliance in Health Care. Baltimore, Md: Johns Hopkins University Press, 1979.

7. Lin IF, Spiga R, Fortsch W. Insight and adherence to medication in chronic schizophrenics. J Clin Psychiatry 1979; 40: 430-432

8. Conrad P. The meaning of medications: Another look at compliance. Soc Sci Med 1985; 20(1): 29-37.

9. Young IL, Zonana HV, Shelper L. Medication non-compliance in schizophrenia: codification and update. Bull Am Academy Psychiatr Law 1986; 14: 105-1 22.

10. Gillis LS, Trollip D, Jakoet A, Holden T. Non-compliance with psychotropic medication S Afr Med J 1987; 72(1 1): 602-606

11. Koch A, Gillis LS. Non-attendance of psychiatric outpatients. S Afr MedJ 1991; 80(9): 289-291.

12. Bebbington PE. The content and context of compliance. Int Clin Psychopharmacol 1995: 9(5): 41-50

13. MacPherson $R$, Jerrom B, Hughes A. Relationship between insight, educational background and cognition in schizophrenia. BrJ Psychiatry 1996; 168: $718-722$

14. Kane JM. What can we achieve by implementing a compliance-improvement programme? Int Clin Psychopharmacol 1997; 12(1): 543-546.

15. Nageotte C, Sullivan G, Duan N, Camp PL. Medication compliance among the seriously mentally ill in a public mental health system. Soc Psychiatry Psychiatr Epidemiol 1997; 32: 49-56.

16. Ruscher SM, de Wit R, Mazmanian D. Psychiatric patients' attitudes about medication and factors affecting non-compliance. Psych Serv 1997; 48(1): 82-85.

17. Thornicroft $G$, Tansella M. Components of a modern mental health service: a pragmatic balance of community and hospital care: overview of systematic evidence. BrJ Psychiatry 2004; 185: 283-290.

18. Swartz L, MacGregor H. Integrating services, marginalising patients: psychiatric patients and primary health care in South Africa. Transcultural Psychiatry 2000; 39(2): 155-172. 